\title{
Robotic versus fluoroscopy-guided pedicle screw insertion for metastatic spinal disease: a matched-cohort comparison
}

\author{
Volodymyr Solomiichuk, MD, ${ }^{1}$ Julius Fleischhammer, MD, ${ }^{2}$ Granit Molliqaj, MD, ${ }^{3}$ Jwad Warda, MD, \\ Awad Alaid, MD, ${ }^{1}$ Kajetan von Eckardstein, MD, ${ }^{1}$ Karl Schaller, MD, ${ }^{3}$ Enrico Tessitore, MD, ${ }^{3}$ \\ Veit Rohde, MD, ${ }^{1}$ and Bawarjan Schatlo, MD'1
}

Departments of ${ }^{1}$ Neurosurgery and ${ }^{2}$ Neuroradiology, Georg-August-University of Göttingen, Germany; and ${ }^{3}$ Department of Neurosurgery, University Hospital Geneva, Université de Genève, Faculté de Médecine, Geneva, Switzerland

OBJECTIVE Robot-guided pedicle screw placement is an established technique for the placement of pedicle screws. However, most studies have focused on degenerative disease. In this paper, the authors focus on metastatic spinal disease, which is associated with osteolysis. The associated lack of dense bone may potentially affect the automatic recognition accuracy of radiography-based surgical assistance systems. The aim of the present study is to compare the accuracy of the SpineAssist robot system with conventional fluoroscopy-guided pedicle screw placement for thoracolumbar metastatic spinal disease.

METHODS Seventy patients with metastatic spinal disease who required instrumentation were included in this retrospective matched-cohort study. All 70 patients underwent surgery performed by the same team of experienced surgeons. The decision to use robot-assisted or fluoroscopy-guided pedicle screw placement was based the availability of the robot system. In patients who underwent surgery with robot guidance, pedicle screws were inserted after preoperative planning and intraoperative fluoroscopic matching. In the "conventional" group, anatomical landmarks and anteroposterior and lateral fluoroscopy guided placement of the pedicle screws. The primary outcome measure was the accuracy of screw placement on the Gertzbein-Robbins scale. Grades A and B (<2-mm pedicle breach) were considered clinically acceptable, and all other grades indicated misplacement. Secondary outcome measures included an intergroup comparison of direction of screw misplacement, surgical site infection, and radiation exposure.

RESULTS A total of 406 screws were placed at 206 levels. Sixty-one (29.6\%) surgically treated levels were in the upper thoracic spine (T1-6), 74 (35.9\%) were in the lower thoracic spine, and the remaining $71(34.4 \%)$ were in the lumbosacral region. In the robot-assisted group (Group I; $n=35,192$ screws), trajectories were Grade A or B in 162 (84.4\%) of screws. The misplacement rate was $15.6 \%$ (30 of 192 screws). In the conventional group (Group II; $n=35,214$ screws), $83.6 \%$ (179 of 214) of screw trajectories were acceptable, with a misplacement rate of $16.4 \%$ (35 of 214 ). There was no difference in screw accuracy between the groups (chi-square, 2-tailed Fisher's exact, $p=0.89$ ). One screw misplacement in the fluoroscopy group required a second surgery (0.5\%), but no revisions were required in the robot group. There was no difference in surgical site infections between the 2 groups (Group I, 5 patients [14.3\%]; Group II, 8 patients [22.9\%]) or in the duration of surgery between the 2 groups (Group I, $226.1 \pm 78.8$ minutes; Group II, $264.1 \pm 124.3$ minutes; $p=0.13$ ). There was also no difference in radiation time between the groups (Group I, $138.2 \pm 73.0$ seconds; Group II, $126.5 \pm 95.6$ seconds; $p=0.61$ ), but the radiation intensity was higher in the robot group (Group I, $2.8 \pm 0.2$ mAs; Group II, $2.0 \pm 0.6$ mAs; $p<0.01$ ).

CONCLUSIONS Pedicle screw placement for metastatic disease in the thoracolumbar spine can be performed effectively and safely using robot-guided assistance. Based on this retrospective analysis, accuracy, radiation time, and postoperative infection rates are comparable to those of the conventional technique.

https://thejns.org/doi/abs/10.3171/2017.3.FOCUS1710

KEY WORDS spine instrumentation; pedicle screw; robotic surgery; spinal fusion; computer-assisted surgery; spinal metastasis 
$\mathrm{M}$ ETASTATIC spine disease accounts for 10\%-30\% of new cancer diagnoses annually. ${ }^{3}$ Radiation therapy, systemic chemotherapy, and surgery are used alone or in combination to treat spinal metastases. Indications for surgery include spinal instability, progressive symptomatic deformities, neurological deficits, and severe pain. ${ }^{10}$ The mainstay of treatment for instability is pedicle screw insertion. Although screw insertion is a standardized procedure, it can be associated with complications, such as screw malpositioning., ${ }^{5,21}$ Most efforts to improve pedicle screw accuracy are based on image guidance with navigation and intraoperative imaging. ${ }^{8}$ For the most part, these techniques appear to be at least comparable to conventional technique in terms of accuracy. ${ }^{10,25}$ Robotic surgery, which has already been used routinely in other specialties, $7,14,16,26$ only recently gained acceptance in spinal surgery.

A commercially available system manufactured by Mazor has been developed to assist the surgeon with the task of pedicle screw placement. ${ }^{12}$ This miniature semiactive robotic system indicates the direction of the pedicle screw trajectory after preoperative planning. The surgeon performs the drilling and screw insertion manually. This robotic system has the advantage of allowing for minimally invasive percutaneous procedures. Previous studies have shown that the use of this system is associated with less radiation exposure. ${ }^{19,23}$ Robot-guided pedicle screw placement is well established for posterior instrumentation of degenerative conditions. ${ }^{11,21}$ Metastatic spinal disease is associated with osteolysis, which may potentially affect the automatic recognition accuracy of surgical assistance systems that are based on cortical bone outlines. The aim of the present study is to compare the accuracy of the SpineAssist robot system (Mazor) with that of conventional fluoroscopy-guided pedicle screw placement for metastatic spinal disease.

\section{Methods}

This retrospective chart review includes patients who underwent posterior instrumentation for instability due to metastatic spinal disease between June 2009 and June 2015. Thirty-five patients underwent surgery with the aid of robot assistance during this period. For a control group, we selected 35 age-matched patients who required elective spinal instrumentation surgery using conventional freehand technique. The parameters of screw accuracy, radiation exposure, and the need for a surgical wound revision were compared between groups. The decision to use robot-assisted or fluoroscopy-guided pedicle screw placement was based the availability of the robot system, not on surgeon preference.

\section{Surgical Technique and Cohorts \\ Robot-Guided Technique}

The surgical technique was performed as described previously. ${ }^{21}$ Briefly, in the robot-guided group (Group I; $\mathrm{n}=35,192$ screws), a CT scan with 3D-compatible data sets was used for planning of pedicle screw trajectories on the SpineAssist system. Using a modified intraoperative fluoroscope and a spinous process clamp or an iliac crest-mounted bridge, we performed intraoperative data

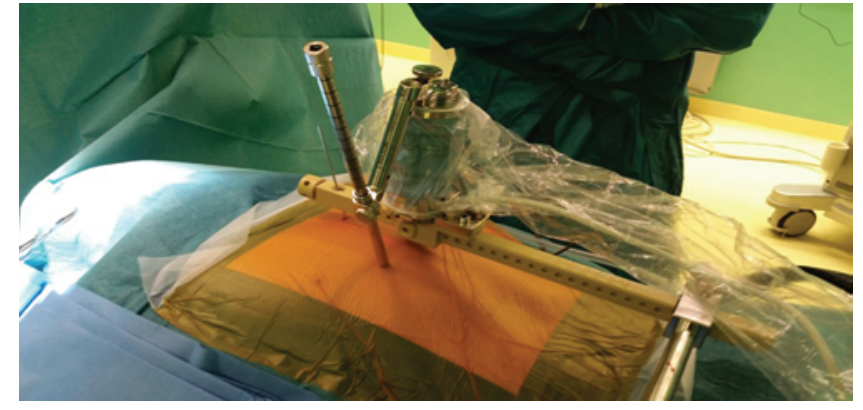

FIG. 1. Photograph of the SpineAssist robot. The miniature robot (blue) is mounted on a bridge. A metal arm and a cannula are attached to the robot. The robot moves to indicate the previously planned trajectory. The surgeon then performs a stab incision and advances the cannula until it reaches a bone surface. Drilling and K-wire insertion are performed. The robot can then move to the next trajectories. Tapping and screw insertion can thus be performed using minimally invasive techniques.

set matching. Drilling and K-wire placement were guided by the robot arm, which was mounted on the bridge (Fig. 1). A minimally invasive technique was used for screw insertion. ${ }^{15}$ Prior to the drilling of the pedicle and during screw insertion, fluoroscopy was used at the discretion of the surgeon.

\section{Fluoroscopy-Guided Surgery}

In the fluoroscopy-guided cohort (Group II; $n=35,214$ screws), the screws were inserted using anatomical landmarks and lateral fluoroscopy guidance through a standard open approach. The spine was prepared subperiostially. After exposure of the posterior elements, the pedicle was probed and instrumented. ${ }^{24}$

\section{Primary Outcome}

Screw accuracy was the primary outcome measure. All patients underwent postoperative 3D CT sagittal. Axial, coronal, and sagittal reconstructions were used to assess the accuracy of screw placement according to the Gertzbein-Robbins scale (from A to E: A, perfect intrapedicular localization; E, > $6 \mathrm{~mm}$ deviation from the ideal intrapedicular trajectory; Fig. 2). ${ }^{24}$ The slice with the largest deviation from the pedicle was chosen for grading. A neuroradiologist (J.F.) was blinded to type of treatment and evaluated all CT scans in both groups. As per radiological convention, screw Grades A and B are clinically acceptable, and screws graded C, D, and E have a significant deviation from the intended trajectory. An "in-out-in" screw placement was not graded differently since retrospective review made it difficult to distinguish between screws that were intentionally placed using this technique and screws that deviated laterally.

\section{Secondary Parameters}

We recorded the need for revision of a misplaced screw or other complications as well as radiation intensity and duration. Moreover, surgical site infections, radiation exposure, and radiation time were recorded.

\section{Statistical Analysis}

The Student t-test, Mann-Whitney U-test, and chi- 

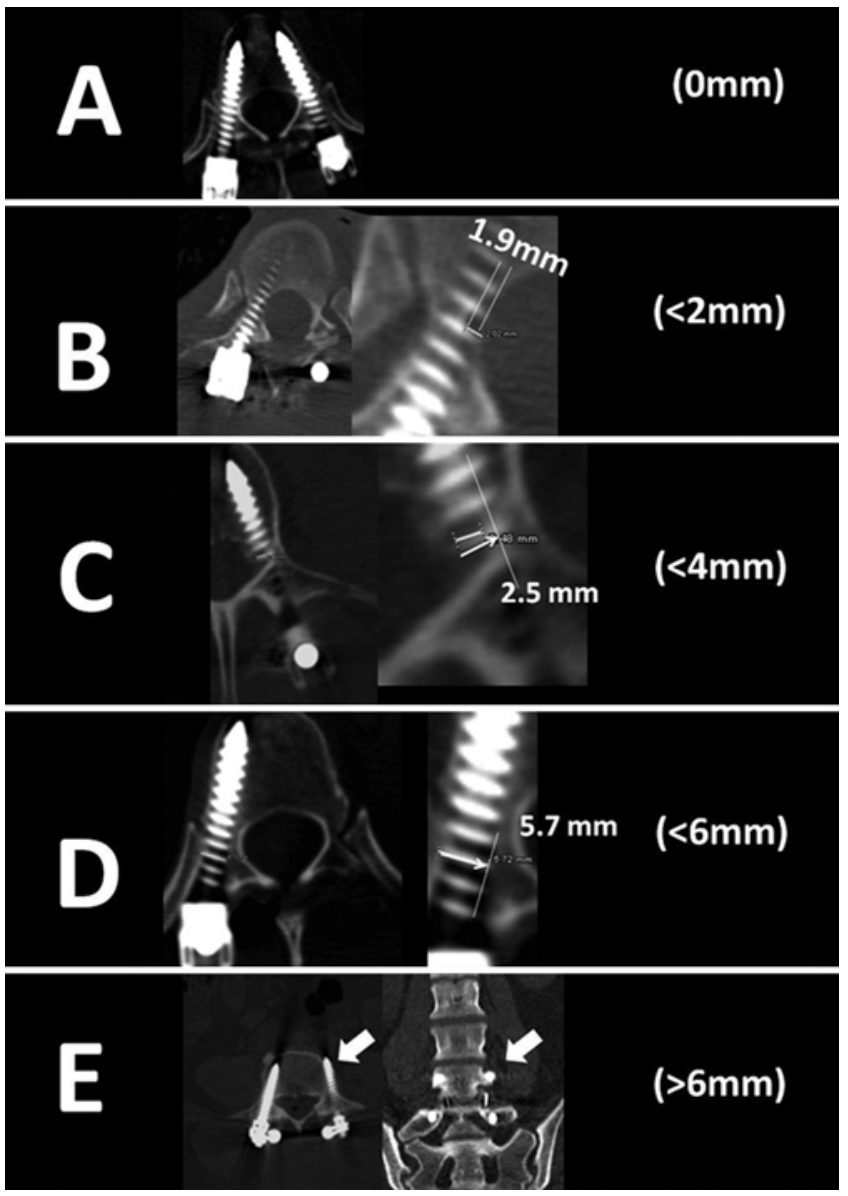

FIG. 2. Gertzbein-Robbins classification. The grading system reflects the deviation of the screw from the "ideal" intrapedicular trajectory. The grades are as follows: Grade A, an intrapedicular screw without breach of the cortical layer of the pedicle; Grade B, a screw that breaches the cortical layer of the pedicle but does not exceed it laterally by more than $2 \mathrm{~mm}$; Grades $C$ and D, penetration of less than 4 and $6 \mathrm{~mm}$, respectively (arrow); and Grade E, screws (arrows) that do not pass through the pedicle or that, at any given point in their intended intrapedicular course, breach the cortical layer of the pedicle in any direction by more than $6 \mathrm{~mm}$. Note that the screw that was graded as $D$ has a deviation of more than $4 \mathrm{~mm}$ from the intrapedicular directory. Modified with permission from Schatlo et al: J Neurosurg Spine 20:636-243, 2014.

square test using 2-tailed Fisher's exact test were used for statistical analysis. Comparisons were considered significantly different at $\mathrm{p}<0.05$.
TABLE 2. Pedicle screw placement accuracy according to the Gertzbein-Robbins classification

\begin{tabular}{ccc}
\hline & \multicolumn{2}{c}{ No. of Screws (\%) } \\
\cline { 2 - 3 } Grade & Robotic Technique & Conventional \\
\hline A & $129(67.2)$ & $136(63.6)$ \\
\hline B & $33(17.2)$ & $43(20.1)$ \\
\hline C & $10(5.2)$ & $18(8.4)$ \\
\hline D & $14(7.3)$ & $9(4.2)$ \\
E & $6(3.1)$ & $8(3.7)$ \\
\hline
\end{tabular}

\section{Results}

\section{Baseline Characteristics}

Baseline parameters did not differ between the robotguided and fluoroscopy-guided groups (Table 1).

\section{Screw Accuracy}

A detailed listing of pedicle screw accuracy grades is provided in Table 2. Overall, in the robot group (Group I), a perfect trajectory (Grade A) was observed in 129 screws $(67.2 \%)$. The remaining screws were graded $\mathrm{B}(\mathrm{n}=33$; $17.2 \%), C(n=10 ; 5.2 \%), D(n=14 ; 7.3 \%)$, and $E(n=6$; $3.1 \%$ ). In the fluoroscopy-guided group (Group II), a completely intrapedicular course (Grade A) was found in 136 $(63.6 \%)$ screws. The remaining screws were graded $\mathrm{B}(\mathrm{n}=$ $43 ; 20.1 \%), C(n=18 ; 8.4 \%), D(n=9 ; 4.2 \%)$, and $E(n=8$; $3.7 \%)$. The comparison of "clinically acceptable" Grade A and B screws (Group I, 162 of 192 [84.4\%]; Group II, 179 of 214 [83.6\%]; $p=0.89$ ) was not statistically different between groups. Screws with a poor trajectory (Grades C-E) occurred with a frequency of 30 of 192 cases (15.6\%) in Group I, and 35 of 214 cases (16.4\%) in Group II, which also failed to reach significance $(\mathrm{p}=0.89)$.

The direction of misplacement was recorded for all screws that were not perfectly intrapedicular (Grades B-E, total $n=141$ ). In 8 cases, the screw was displaced in 2 directions, leading to a total of 149 counts (67 in Group I and 82 in Group II), which therefore exceeds the total number of misplaced screws (Table 3). There were no cranial displacements in either group. Caudal displacement occurred in 5 screws in Group I (7.4\%) and 5 in Group II $(6.1 \%$; $\mathrm{p}=0.76)$, medial displacement in 33 screws in Group I (49.3\%) and in 21 in Group II (25.6\%; p < 0.01), and lateral displacement in 29 of Group I (43.3\%) and 55 of Group

TABLE 1. Baseline characteristics

\begin{tabular}{|c|c|c|c|c|c|c|c|}
\hline \multirow[b]{2}{*}{ Variable } & \multicolumn{2}{|c|}{ Conventional } & \multicolumn{2}{|c|}{ Robotic Technique } & \multicolumn{2}{|c|}{ Total } & \multirow[b]{2}{*}{$p$ Value } \\
\hline & Mean & SD & Mean & SD & Mean & SD & \\
\hline Age, yrs & 62.2 & 11.1 & 63.7 & 10.6 & 65.2 & 10.1 & 0.24 \\
\hline$\%$ male sex & 23 & $65.7 \%$ & 21 & $60.0 \%$ & 44 & $62.9 \%$ & 0.81 \\
\hline No. of screws/case & 6.1 & 2.3 & 5.5 & 2.1 & 5.8 & 2.3 & 0.14 \\
\hline No. of vertebrae & 3.2 & 1.2 & 2.8 & 1.1 & 3.0 & 1.2 & 0.12 \\
\hline Duration of surgery, mins & 264.1 & 124.3 & 226.1 & 78.8 & 245.1 & 105.0 & 0.13 \\
\hline Radiation time, secs & 126.5 & 95.6 & 138.2 & 73.0 & 132.1 & 84.9 & 0.61 \\
\hline Radiation intensity, mAs & 2.0 & 0.6 & 2.8 & 0.2 & 2.7 & 0.4 & $<0.01$ \\
\hline
\end{tabular}


TABLE 3. Screw misplacement rates

\begin{tabular}{lccc}
\hline & \multicolumn{2}{c}{ No. of Screws (\%) } & \\
\cline { 2 - 3 } Misplacement & Robot Technique & Conventional & p Value \\
\hline Upper thoracic & $9(4.7)$ & $18(8.4)$ & 0.82 \\
\hline Lower thoracic & $14(7.3)$ & $15(7.0)$ & 0.54 \\
\hline Lumbosacral & $7(3.6)$ & $2(0.9)$ & 0.18 \\
\hline Total & $30(15.6)$ & $35(16.4)$ & \\
\hline
\end{tabular}

II trajectories (67.1\%; p < 0.01; Figs. 3 and 4). Therefore, screw deviation was more frequently medially directed in the robot group (Group I), while it was more frequently laterally directed in the conventional group (Group II).

\section{Duration of Surgery}

There was no statistically significant difference in duration of surgery between the two groups (Group I, 226.1 \pm 78.8 minutes, Group II, $264.1 \pm 124.3$ minutes; $p=0.13$ ).

\section{Complications}

There was no difference in surgical site infection rates between the groups (Group I, 5 patients [14.3\%]; Group II, 8 patients [22.9\%]; $\mathrm{p}=0.54$ ). All the patients with wound infections underwent wound revision. Neurological injury occurred in 1 case in Group II; displacement of all 4 screws, caused a painful radiculopathy at T-4 due to foraminal impingement without neurological deficit. A second surgery was required to replace the screws, and the radiculopathy resolved. This was the only revision surgery.

\section{Radiation Exposure}

No difference was found in the duration radiation time between the groups (Group I, 138.2 \pm 73.0 seconds; Group II, $126.5 \pm 95.6$ seconds; $p=0.61$ ). Only radiation intensity was higher in the robot group (Group I, $2.8 \pm 0.2 \mathrm{mAs}$; Group II, $2.0 \pm 0.6 \mathrm{mAs} ; \mathrm{p}<0.01)$, which could be a result of high-quality fluoroscopy that was needed for the robot system registration process.

\section{Discussion}

\section{Advances in Accuracy and Safety of Pedicle Screw Placement}

The SpineAssist system is only one of many devices designed to improve pedicle screw accuracy and thus pa-

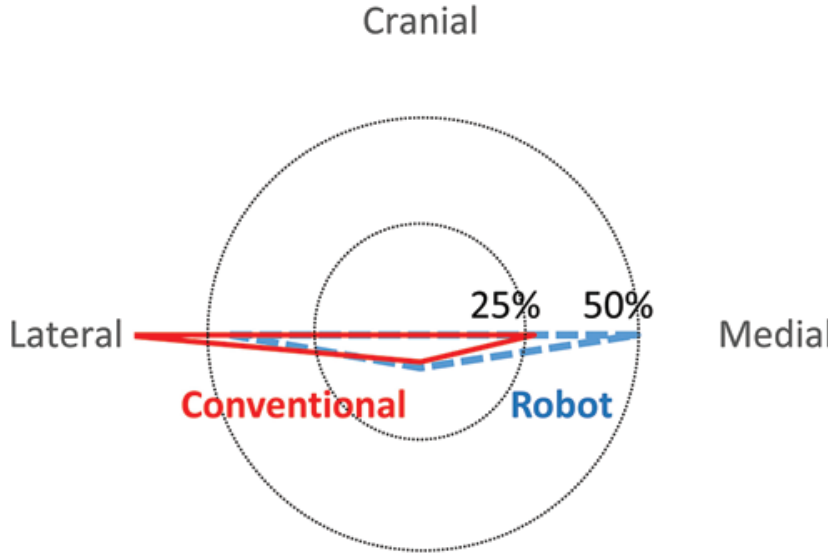

\section{Caudal}

FIG. 4. Radar chart showing the proportion (\%) of screw displacement in the robot (blue) and conventional group (red). There were no cranial displacements in either group. Caudal displacement occurred in 5 screws in Group I (7.4\%) and 5 in Group II (6.1\%; $p=0.76)$, medial displacement in 33 screws in Group I (49.3\%) and 21 in Group II (25.9\%; $p<0.01)$, and lateral displacement in 29 in Group I (43.3\%) and 55 in Group II (67.1\%; $p<0.01)$. Therefore, screw deviation was more frequently medially directed in the robot group (Group I), while it was more frequently lateral in the conventional group (Group II).

tient safety. Image-guided systems are useful in helping achieve better implant accuracy and reduce the need for surgical revisions. ${ }^{1,22}$ Additional methods such as conductivity measurement devices that detect cortical defects have equally proven useful in this respect. ${ }^{2}$

Malposition rates with conventional screw placement can be significant. ${ }^{6}$ However, the term "malposition" is not well defined and depends on the various grading systems, which are available to evaluate screw position. The majority of pedicle screws with minor breach of pedicle cortex may still have excellent biomechanical properties. Thus, one may define a malpositioned screw as a screw with the potential of clinically apparent neurological or vascular impairment or biomechanical insufficiency. Consequently, manifestations of screw malposition include instability, fractures, injury to dura, vessels, or nervous structures. ${ }^{6}$ In contrast to mere malposition on imaging, the incidence of neurological complications of pedicle screw placement in the literature is extremely low. ${ }^{4}$

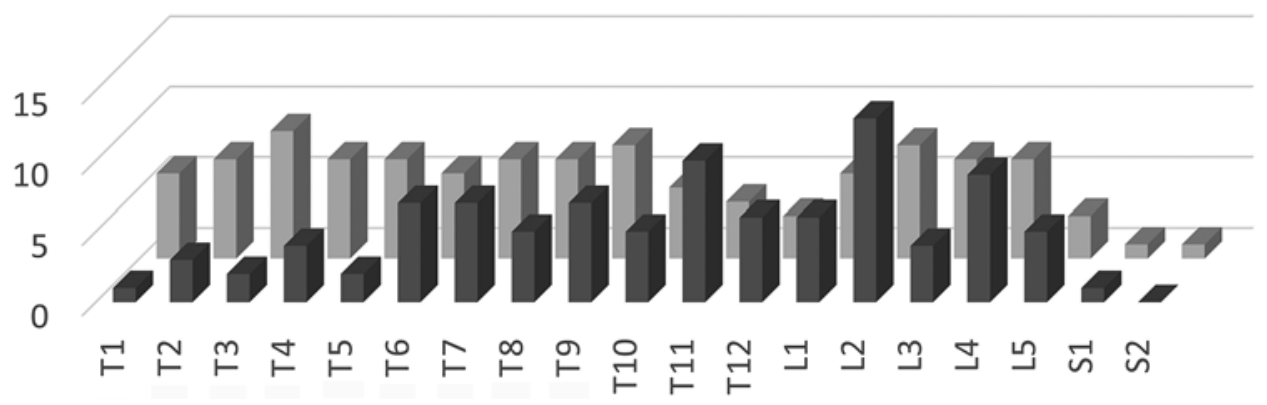

FIG. 3. Distribution of operated levels in both (robot and conventional) groups. This graph depicts the number of surgeries (y-axis) performed on vertebral segments (x-axis) in the robot (dark gray) and conventional (light gray) groups. 
In this study, we present data assessing the utility and feasibility of robot-assisted insertion of pedicle screws in patients with metastatic spinal disease. This is of particular relevance since the registration process that is at the heart of the robot device's image recognition and segmentation system relies on the contours of vertebrae observed on lateral and anteroposterior radiographs. It may therefore have been possible that accuracy of pedicle screw insertion in cases of degeneration with intact bone is excellent, while the accuracy of the robotic system in patients with osteolytic phenomena is reduced. Despite this concern, we found that the accuracy of pedicle screw insertion with the aid of a robotic guidance system is similar to that of the fluoroscopy-guided open conventional technique. This finding is based on screws placed in the thoracic as well as the lumbar spine.

\section{Accuracy}

The rate of accurate screw placement with the aid of a Mazor robotic system ranges from $85 \%{ }^{18}$ to $98.5 \%,{ }^{17}$ depending on the report and the generation of the system used. In addition to the extent of pedicle breach, robotic surgery has been found to be more reliable than a freehand technique in achieving sufficient screw convergence and avoiding facet breach. ${ }^{12}$ Our data are novel in that they add a large set of data on thoracic instrumentation, which, until now, has received little attention in previous reports. Thoracic screws placed with the aid of the robotic system had similar rates of pedicle breach compared with those placed using conventional technique. No revision surgeries were noted in the robot group, while revision was necessary because of nerve root impingement caused by a misplaced pedicle screw in the conventional group. As in previous studies, the low rate of revision surgeries makes it difficult to identify significant differences between the groups. ${ }^{21}$ The direction of screw deviation was previously reported to be more lateral in robot-guided screws compared with conventional screw placement. ${ }^{21}$ Interestingly, we found that the opposite was true in our cohort, where screw misplacements with the robot were more frequently medial (albeit without the need for revision), while screw placements were more frequently lateral in the conventional group. This discrepancy to other studies may be twofold. First, a higher proportion of thoracic screws were placed in our cohort than in cohorts of other robot studies. In addition, lateral misplacement, which may be radiologically graded as misplacement, may simply be the result of an intended in-out-in technique in the conventional group. Second, the medially and ventrally directed slope of the costotransverse process may account for medial screw deviations in the robot group. While lateral misplacements were more frequent using robots in the lumbar spine ${ }^{18}$ our study found more frequent lateral misplacements in the freehand group. One potential explanation is that the Gertzbein-Robbins grading system does not exempt "inout-in screws" from being considered misplacements. There is a methodological difficulty with retrospectively assessing whether a screw was laterally "misplaced" or intentionally placed using the in-out-in technique. Although the study design is retrospective, we believe that the independent and blinded review of screw placement allows conclusions regarding the utility of robotic assistance. This study is based on the SpineAssist device, and therefore our conclusions should only be applied to the use of this specific system.

\section{Radiation Exposure}

In degenerative cases, use of robotic technology is associated with less radiation exposure to the patient and personnel..$^{19}$ In the present study, duration of radiation exposure was similar in both the conventional fluoroscopy and robotic surgery groups. This constitutes a new finding that merits further exploration. In a setting where accuracy of screw placement is similar between groups, the main expectation of a surgical assistance system would be a reduction in radiation exposure. The major difference setting our study apart from previous reports ${ }^{12}$ is that it is based on a uniform cohort of patients suffering from instability due to osteolytic metastatic disease. Because of osteolytic phenomena, problems with registration or the need for repeated verification of trajectories suggested by the robot arm may have led to increased intraoperative use of the $\mathrm{C}$-arm compared with degenerative cases. The advantage of a reduced exposure to radiation when using robotic technology may therefore be limited in the setting of osteolytic spinal disease. Moreover, our experience showed that automated registration by the robot software is more difficult in the thoracic spine, leading to increased times of surgery and the need for repeated imaging to improve registration accuracy. The robot trajectory can be affected by surgeon-related factors such as insufficient experience ${ }^{20}$ or registration issues. ${ }^{9,18}$

\section{Pearls for the Optimal Use of the Robotic System}

One of the supposed advantages of a robot system is its independence from a surgeon's experience. Our data suggest that, despite this claim, surgeons may encounter difficulties during the first few cases, at least with the use of the first-generation system. ${ }^{20}$ As with all auxiliary techniques, robotic assistance systems will initially add some extra time to the surgery. To optimize the robot experience, surgeon and operating room personnel can undergo training, e.g., in cadaver courses or by visiting a center that already uses the system. Moreover, a dry run prior to the first use of the system may help the surgeon obtain the necessary familiarity with its features. During patient positioning, cables and radiopaque material should not be in the way of the C-arm. This simple step avoids having to deal with image processing problems when the anteroposterior and oblique lateral registration shots are obtained. Planning screw placement using the software is intuitive. Attention should be paid to place the entry point of a screw on a flat surface to avoid having the fine-tipped cannula slide off a bone spur. The planning software allows the alignment of the screw heads. This feature helps the surgeon obtain information on rod shape and length. Rigid fixation of the robot bridge to the patient's spine or iliac crest is verified before and after registration. The use of bed-mounted frames that are not attached to the patient is reserved for exceptionally simple 1-level procedures. Finally, the stab incisions should be sufficiently large and include the fascia to avoid soft-tissue pressure on the cannula. Routine use of fluoroscopy during the pedicle cannulation process is 
advisable. In rare instances, the robotic arm may appear to indicate a trajectory that does not match the preoperative plan. Such a mismatch should prompt a systematic reappraisal of the robot setup, the software plan, and the rigidity of the bone-mounted bridge. Usually, these instances are resolved after checking through these items. Rarely, robot guidance has to be converted into conventional minimally invasive or open technique.

\section{Complications After Instrumentation for Metastatic Disease of the Spine}

Complication rates from posterior instrumentation range from $10 \%$ to $52 \%{ }^{3}$ and can be classified as surgical (e.g., wound infections, CSF fistulas), hardware related (broken, misplaced, migrated), medical (e.g., pneumonia, deep venous thrombosis, myocardial infarct), and neurological (i.e., new sensory or motor deficit). ${ }^{13}$ One of the most problematic surgical complications is wound infection, which is seen in up to $15 \%$ of cases. ${ }^{3}$ The present study showed no difference in surgical site infection rates between fluoroscopy and robotic surgery groups, alleviating concerns that the added technical effort to perform posterior instrumentation may lead to an increased rate of complications.

\section{Limitations of the Cohort Comparison}

Herein, we compared 2 matched cohorts in which either robot assistance or fluoroscopic guidance was used. One limitation is that the control group underwent surgery using an open technique, while the percutaneous robot group underwent a minimally invasive-type surgery. The aim of the present analysis was to compare accuracy, which is a radiological assessment. While no difference was found in placement accuracy between groups, our data do not allow us to conclude that the compared groups are equivalent in terms of surgical outcome. Despite an attempt to match the groups, minor heterogeneities were found: the number of screws and instrumented segments and the number of instrumented levels was higher in the fluoroscopy-guided group (Group II) than in the robot cohort (Group I), which may equally have contributed to a more difficult surgery in the fluoroscopy-guided group. Hypothetically, without this heterogeneity, the number of well-positioned screws in the fluoroscopy group may have been somewhat higher.

\section{Conclusions}

Pedicle screw placement for metastatic disease in the thoracolumbar spine can be performed effectively and safely using robot-guided assistance, but accuracy, radiation time, and postoperative infection rates are similar to conventional technique.

\section{References}

1. Beck M, Mittlmeier T, Gierer P, Harms C, Gradl G: Benefit and accuracy of intraoperative 3D-imaging after pedicle screw placement: a prospective study in stabilizing thoracolumbar fractures. Eur Spine J 18:1469-1477, 2009

2. Bolger C, Kelleher MO, McEvoy L, Brayda-Bruno M, Kaelin A, Lazennec JY, et al: Electrical conductivity measurement: a new technique to detect iatrogenic initial pedicle perforation. Eur Spine J 16:1919-1924, 2007
3. Dunning EC, Butler JS, Morris S: Complications in the management of metastatic spinal disease. World J Orthop 3:114-121, 2012

4. Faraj AA, Webb JK: Early complications of spinal pedicle screw. Eur Spine J 6:324-326, 1997

5. Gaines RW Jr: The use of pedicle-screw internal fixation for the operative treatment of spinal disorders. J Bone Joint Surg Am 82-A:1458-1476, 2000

6. Gautschi OP, Schatlo B, Schaller K, Tessitore E: Clinically relevant complications related to pedicle screw placement in thoracolumbar surgery and their management: a literature review of 35,630 pedicle screws. Neurosurg Focus 31(4):E8, 2011

7. Gutt CN, Oniu T, Mehrabi A, Kashfi A, Schemmer P, Büchler MW: Robot-assisted abdominal surgery. Br J Surg 91:13901397, 2004

8. Holly LT, Foley KT: Intraoperative spinal navigation. Spine (Phila Pa 1976) 28 (15 Suppl):S54-S61, 2003

9. Hu X, Lieberman IH: What is the learning curve for roboticassisted pedicle screw placement in spine surgery? Clin Orthop Relat Res 472:1839-1844, 2014

10. Hu X, Scharschmidt TJ, Ohnmeiss DD, Lieberman IH: Robotic assisted surgeries for the treatment of spine tumors. Int J Spine Surg 9:1, 2015

11. Kantelhardt SR, Martinez R, Baerwinkel S, Burger R, Giese A, Rohde V: Perioperative course and accuracy of screw positioning in conventional, open robotic-guided and percutaneous robotic-guided, pedicle screw placement. Eur Spine J 20:860-868, 2011

12. Kim HJ, Jung WI, Chang BS, Lee CK, Kang KT, Yeom JS: A prospective, randomized, controlled trial of robot-assisted vs freehand pedicle screw fixation in spine surgery. Int $\mathbf{J}$ Med Robot [epub ahead of print], 2016

13. Klimo P Jr, Schmidt MH: Surgical management of spinal metastases. Oncologist 9:188-196, 2004

14. Lang MJ, Greer AD, Sutherland GR: Intra-operative robotics: NeuroArm. Acta Neurochir Suppl 109:231-236, 2011

15. Lieberman IH, Togawa D, Kayanja MM, Reinhardt MK, Friedlander A, Knoller N, et al: Bone-mounted miniature robotic guidance for pedicle screw and translaminar facet screw placement: Part I-Technical development and a test case result. Neurosurgery 59:641-650, 2006

16. Nakamura N, Sugano N, Nishii T, Kakimoto A, Miki H: A comparison between robotic-assisted and manual implantation of cementless total hip arthroplasty. Clin Orthop Relat Res 468:1072-1081, 2010

17. Onen MR, Simsek M, Naderi S: Robotic spine surgery: a preliminary report. Turk Neurosurg 24:512-518, 2014

18. Ringel F, Stüer C, Reinke A, Preuss A, Behr M, Auer F, et al: Accuracy of robot-assisted placement of lumbar and sacral pedicle screws: a prospective randomized comparison to conventional freehand screw implantation. Spine (Phila Pa 1976) 37:E496-E501, 2012

19. Roser F, Tatagiba M, Maier G: Spinal robotics: current applications and future perspectives. Neurosurgery 72 (Suppl 1): $12-18,2013$

20. Schatlo B, Martinez R, Alaid A, von Eckardstein K, Akhavan-Sigari $\mathrm{R}$, Hahn A, et al: Unskilled unawareness and the learning curve in robotic spine surgery. Acta Neurochir (Wien) 157:1819-1823, 2015

21. Schatlo B, Molliqaj G, Cuvinciuc V, Kotowski M, Schaller $\mathrm{K}$, Tessitore E: Safety and accuracy of robot-assisted versus fluoroscopy-guided pedicle screw insertion for degenerative diseases of the lumbar spine: a matched cohort comparison. J Neurosurg Spine 20:636-643, 2014

22. Scheufler KM, Franke J, Eckardt A, Dohmen H: Accuracy of image-guided pedicle screw placement using intraoperative computed tomography-based navigation with automated referencing. Part II: thoracolumbar spine. Neurosurgery 69:1307-1316, 2011 
23. Sensakovic WF, O’Dell MC, Agha A, Woo R, Varich L: CT radiation dose reduction in robot-assisted pediatric spinal surgery. Spine (Phila Pa 1976) [epub ahead of print], 2016

24. Steffee AD, Sitkowski DJ: Posterior lumbar interbody fusion and plates. Clin Orthop Relat Res 227:99-102, 1988

25. Tjardes T, Shafizadeh S, Rixen D, Paffrath T, Bouillon B, Steinhausen ES, et al: Image-guided spine surgery: state of the art and future directions. Eur Spine J 19:25-45, 2010

26. Wiklund NP: Technology Insight: surgical robots-expensive toys or the future of urologic surgery? Nat Clin Pract Urol 1:97-102, 2004

\section{Disclosures}

Dr. Tessitore reports that he is a consultant for Mazor.

\section{Author Contributions}

Conception and design: Schatlo, Solomiichuk, von Eckardstein, Schaller. Acquisition of data: Solomiichuk, Fleischhammer, Tessitore. Analysis and interpretation of data: Schatlo, Solomiichuk, Fleischhammer, Molliqaj, Alaid, Tessitore. Drafting the article: Solomiichuk. Critically revising the article: Schatlo, Rohde. Reviewed submitted version of manuscript: Schatlo, Molliqaj, von Eckardstein, Schaller, Tessitore, Rohde. Approved the final version of the manuscript on behalf of all authors: Schatlo. Statistical analysis: Schatlo, Solomiichuk, Molliqaj, Warda, Alaid. Administrative/technical/material support: Warda. Study supervision: Schatlo, Rohde.

\section{Correspondence}

Bawarjan Schatlo, Department of Neurosurgery, Universitätsklinik Göttingen Robert-Koch-Str. 40, Göttingen 37075, Germany.email: bawarjan.schatlo@med.uni-goettingen.de. 